\title{
Patlak Plot
}

National Cancer Institute

\section{Source}

National Cancer Institute. Patlak Plot. NCI Thesaurus. Code C80353.

A graphical analysis technique based on a two compartment model that uses linear regression to identify and analyze pharmacokinetics problems involving irreversible uptake in one compartment. 